\title{
PDE Control of Heat Exchangers by Input-Output Linearization Approach
}

\author{
Ahmed MAIDI $^{a}$, and Jean-Pierre CORRIOU ${ }^{b}$ \\ ${ }^{a}$ Laboratoire de Conception et Conduite des Systèmes de Production, \\ Université Mouloud MAMMERI, 15000 Tizi-Ouzou, Algérie. \\ ${ }^{b}$ Reaction and Process Engineering Laboratory, \\ UMR 7274-CNRS, Lorraine University, ENSIC \\ 1, rue Grandville, BP 20451, 54001 Nancy Cedex, France.
}

\begin{abstract}
Heat exchangers are widely used in industry for cooling or heating fluids. Their tight control is important for optimal energy management. The dynamical behavior of heat exchangers is described by a set of coupled partial differential equations describing the profiles of internal and external temperatures, which makes their control a challenging problem. The present chapter discusses the geometric control of both counter-current and co-current flow heat exchangers. The simplest control approach would lie on a priori discretization of the PDEs resulting in a set of ODEs that can be tackled by traditional control laws but that does not preserve the fundamental control properties of the heat exchanger (controllability, observability and stability). In the following, the PDEs are directly used to derive the control laws and the discretization is performed a posteriori only for simulation needs. The
\end{abstract}


design of control laws is based on the concept of characteristic index in the framework of input-output linearization and the closed loop stability is demonstrated using some tools from semigroup theory. The control objective consists in controlling the outlet cold fluid temperature by manipulating the inlet hot fluid temperature. Then, robustness with respect to parameter uncertainty and practical implementation are discussed and a general control strategy is introduced. Both tracking and disturbance rejection performances of the developed control laws are assessed by numerical simulation.

Keywords: Distributed parameter system, partial differential equation, heat exchanger, boundary control, geometric control, characteristic index, input-output linearization.

\section{Introduction}

Heating or cooling of liquid and gaseous fluids are two operations involved in many engineering applications. These operations are achieved using heat exchangers that transfer heat from a hot fluid to a cooler one (Al-Dawery et al., 2012; Kakaş and Liu, 2002). Heat exchangers can be classified according to the geometry construction (tubes and plates), to the transfer processes (direct and indirect contacts), to the flow arrangement (counter, parallel and cross-flows) and to the fluid phase (liquid-liquid and gas-liquid) (Kakaş and Liu, 2002). Tube heat exchangers are widely used in industry due to their ability to ensure a wide range of operating temperatures (Al-Dawery et al., 2012; Fratczak et al., 2018).

In a heat exchanger, convection is usually the dominant form of heat transfer compared to diffusion, which is negligible (Arbaoui et al., 2007; Friedly, 1972; Ray and Ogunnaike, 1994). Thus, the dynamic behavior of a heat exchanger is often captured by two linear partial differential equations (PDEs) of hyperbolic type with one spatial variable. These equations, obtained by writing the energy balances separately for both fluids, are weakly coupled via the source terms (difference of temperatures between the two fluids) (Friedly, 1972; Ray and Ogunnaike, 1994). Each PDE describes the spatio-temporal evolution of the temperature of the related fluid. Thus, a heat exchanger is typically an example of a $2 \times 2$ (2 equations with 2 dependent variables, i.e. time and space) hyperbolic system issued from balance laws (Bartecki, 2016).

Heat exchangers are characterized by two flow rates and two inlet temperatures of fluids. These variables can be used as manipulated variables to control the fluid temperatures at the outlet (Maidi et al., 2009). Note that the fundamental control-theoretic properties (controllability, observability and 
stability) depend on the choice of manipulated variables (Alotaibi et al., 2004). By manipulating the inlet temperatures, the heat exchanger is a linear distributed parameter system (DPS), consequently control-theoretic properties can be easily analyzed using the powerful semi-group theory (Grabowski, 2007; Kunimatsu, 1998; Sano, 2007). In the case of flow rate manipulation, the heat exchanger is a nonlinear (bilinear) DPS (Bühler and Franke, 1980) and both control design and control-theoretic properties analysis are difficult tasks.

Essentially, to deal with control design for heat exchangers, two main approaches (Christofides, 2001; Ray, 1989), indirect or direct, are possible. The indirect approach (early lumping) consists in reducing the PDEs to a set of ordinary differential equations (ODEs) by approximating either the PDEs themselves (by discretization) or their solutions (Li and Qi, 2010). This approach allows to exploit various well established linear control theories of lumped parameter systems (LPSs). Nevertheless, the indirect approach masks the distributed nature of the heat exchanger and often the fundamental controltheoretic properties are lost due to the approximation process (Christofides, 2001; Ray, 1989; Singh and Hahn, 2007). In addition, this approach leads to a high dimensional controller with limited performance and difficult practical implementation issues (Christofides and Daoutidis, 1996). The direct approach (late lumping) consists in using directly the PDEs model to design the controller without any previous approximation. The direct approach preserves control-theoretic properties of the heat exchanger and leads to an infinite dimensional controller that is of distributed nature, which enhances the control quality (Christofides and Daoutidis, 1996; Maidi et al., 2009, 2010). Nevertheless, the practical implementation needs the use of an observer to reconstruct the whole state from the accessible measurements (Maidi et al., 2009, 2010).

Heat exchanger control has been extensively investigated in the literature and many control design techniques are proposed. The heat exchanger control literature reveals that most contributions deal with the control of heat exchanger by the indirect approach whereas the direct approach is less addressed. This can be explained by the fact that designing the controller by using directly the PDEs model is a difficult task.

In the following, different control methods of heat exchangers using both indirect and direct approaches are briefly reviewed. First, consider control methods based on the approximate or reduced model. Malleswararao and Chidambaram (1992) proposed a model reference non-linear controller with PID actions. Fischer et al. (1998) and Mazinan and Sadati (2010) developed fuzzy predictive controllers. 
Fuzzy control and neural network approaches have been adopted by Al-Dawery et al. (2012) and Bittanti and Piroddi (1997); Vasičkaninová et al. (2011), respectively. Based on the convexity property, Abdelghani-Idrissi et al. (2001) developed a predictive functional controller. Using an approximate first order model, an adaptive version of a predictive functional controller is implemented by Arbaoui et al. (2007). A control strategy combining neural network predictive and fuzzy controllers is proposed by Vasičkaninová and Bakošová (2015). A sliding mode controller is developed in Almutairi and Zribi (2012). Other advanced robust control methods has also proposed by Dulău et al. (2015); Gauthier and Xu (1991); Kanoh et al. (1997); Vasičkaninová and Bakošová (2016) and Vasičkaninová et al. (2018).

Considering the direct approach, few contributions have been reported in the literature. Hanczyc and Palazoglu (1995) designed a sliding mode control based on the equivalent LPS model obtained using the method of characteristics. Robust control theory has been generalized to DPSs by Pohjolainen and Lätti (1983), thanks to the operators theory, and a flow adaptive robust controller is developed. Using semigroup theory, Sano (2015) proposed a residual mode filter-based controller to enhance the stability under a boundary actuation and boundary sensing. In Aulisa et al. (2016), the nonlinear tracking problem is solved using velocity control configuration. Fratczak et al. (2018) developed a distributed parameter balance-based adaptive controller and performed a practical validation on a laboratory heat exchanger. Boundary control based on the input-output linearization approach has been solved by Maidi et al. $(2009,2010)$.

Geometric control is an interesting late lumping approach that is well adapted for control problem of DPSs (Christofides and Daoutidis, 1996; Maidi and Corriou, 2011b, 2014a,b; Shang et al., 2005; $\mathrm{Wu}$ and Liou, 2001). The present chapter is devoted to the direct control design of geometric control laws for counter-current and co-current heat exchangers. The chapter starts with the control problem formulation. Then, the design approaches based on the PDEs model are presented. The stability and practical implementation issues of the developed control laws are also discussed. Finally, the performance of the presented control laws are evaluated via numerical simulation. The chapter ends with a conclusion.

\section{Heat exchanger control problem}

The considered heat exchanger is a double pipe used to heat the cold fluid circulating in the internal tube by means of the hot fluid circulating in the external tube as depicted in Figure 1 . In the counter- 
current heat exchanger, the hot and cold fluids flow in opposite directions (Fig. 1a) while in the case of a co-current heat exchanger (Fig. 1b), both fluids flow in the same direction. Under some reasonable assumptions (Arbaoui et al., 2007; Friedly, 1972; Ray and Ogunnaike, 1994), the dynamical behavior of this double pipe heat exchanger is described by the following $2 \times 2$ hyperbolic of balance laws (Friedly, 1972):

$$
\begin{aligned}
\frac{\partial T_{h}(z, t)}{\partial t} & =-\vartheta_{h} \frac{\partial T_{h}(z, t)}{\partial z}+\alpha_{h}\left(T_{c}(z, t)-T_{h}(z, t)\right) \\
\frac{\partial T_{c}(z, t)}{\partial t} & =\beta \vartheta_{c} \frac{\partial T_{c}(z, t)}{\partial z}+\alpha_{c}\left(T_{h}(z, t)-T_{c}(z, t)\right) \\
T_{h}\left(\frac{(1+\beta)}{2} l, t\right) & =\frac{(1-\beta)}{2} T_{h_{0}}(t)+\frac{(1+\beta)}{2} T_{h_{l}}(t) \\
T_{c}(0, t) & =T_{c_{0}}(t) \\
T_{h}(z, 0) & =T_{h}^{*}(z) \\
T_{c}(z, 0) & =T_{c}^{*}(z)
\end{aligned}
$$

In the heat exchanger model (1)-(6), $t \in \mathbb{R}^{+}$and $z \in \Omega$ are the time and space independent variables, respectively. $l$ is the length of the heat exchanger and $\Omega=[0, l]$ is the space domain. $T_{h}$ and $T_{c}$ denote the temperatures of the hot and cold fluids, respectively, and $T_{h}^{*}$ and $T_{c}^{*}$ are their initial temperature profiles. $T_{c_{0}}$ is the inlet temperature of the cold fluid. $T_{h_{0}}$ and $T_{h_{l}}$ denote the inlet temperatures of the hot fluid for the co-current and counter-current heat exchangers, respectively. $\alpha_{h}$ and $\alpha_{c}$ are the heat transfer coefficients, $\vartheta_{h}$ and $\vartheta_{c}$ are the flow rates of the fluids. $\beta$ is a constant parameter that depends on the directions of the fluids, that is, $\beta=1$ for a counter-current heat exchanger whereas for a co-current one, $\beta=-1$. Equation (3) means that $T_{h}(l, t)=T_{h_{l}}(t)$ for a counter-current heat exchanger and $T_{h}(0, t)=T_{h_{0}}(t)$ for a co-current one. Thus, it allows to use a single model for both counter-current and co-current heat exchangers.

In the sequel, $L^{2}(\Omega)$ denotes the space of square-integrable functions (Atkinson and Han, 2009), defined on the domain $\Omega$, and we assume that the state vector $T(z, t)=\left[T_{h}(z, t) T_{c}(z, t)\right]^{T} \in \mathcal{H}$ with 
the state space $\mathcal{H}=L^{2}(\Omega) \oplus L^{2}(\Omega)$ endowed with the inner product

$$
\langle f(z), g(z)\rangle_{\mathcal{H}}=\int_{0}^{l} f^{T}(z) g(z) d z, \quad \forall f(z)=\left[\begin{array}{c}
f_{1}(z) \\
f_{2}(z)
\end{array}\right], g(z)=\left[\begin{array}{c}
g_{1}(z) \\
g_{2}(z)
\end{array}\right] \in \mathcal{H}
$$

and the norm

$$
\|f(z)\|_{\mathcal{H}}^{2}=\langle f(z), f(z)\rangle_{\mathcal{H}}
$$

The control problem of the heat exchanger consists in regulating the outlet temperature of the cold fluid towards a desired value. This objective can be achieved by manipulating either the inlet temperature or the flow rate of the hot fluid (Maidi et al., 2009). Nevertheless, controllability is a crucial question to be addressed before designing a controller for a heat exchanger because the reachable temperature depends on the inlet temperature and the flow rate, which are practically constrained (Alotaibi et al., 2004; Sano, 2007). As mentioned above, by manipulating the inlet temperature, the heat exchanger is a linear DPS and the controllability property can be assessed using some tools from the powerful semi-group theory (Sano, 2007). On other hand, by manipulating the flow rate, the heat exchanger is a bilinear DPS (a particular class of nonlinear systems) and the controllability analysis constitutes an open problem. The controllability of heat exchangers is less investigated in the literature. The conducted studies have shown that heat exchangers are controllable with the inlet temperatures and less controllable with flow rates (Alotaibi et al., 2004).

In the following, the studied problem is the control of the output $T_{c_{l}}(t)$ defined as the outlet temperature of the cold fluid, that is,

$$
T_{c_{l}}(t)=T_{c}(l, t)
$$

by manipulating the inlet temperature of the hot fluid, that is, by manipulating either $T_{h_{0}}(t)$ (case of the co-current) or $T_{h_{l}}(t)$ (case of the counter-current). Thus, the inlet temperature of the cold fluid $T_{c_{0}}(t)$ and both flow rates $\vartheta_{h}$ and $\vartheta_{c}$ are assumed to be disturbances.

Note that, according to the studied control problem, it follows that the actuator and the sensor are collocated (placed at the same boundary $z=0$ ) in the case of co-current heat exchanger whereas for the counter-current heat exchanger they are anti-collocated (placed at opposite boundaries, i.e. $z=0$ 
and $z=l$ ). In both cases, the heat exchanger is a linear DPS and the control problem can be tackled easily in the framework of geometric control. A summary of these characteristics is given in Table 1.

\section{Geometric control of heat exchanger}

Design of geometric control laws for DPSs is based on the concept of the characteristic index, which constitutes a generalization of the concept of relative order (Isidori, 1995; Kravaris and Kantor, 1990a) of a LPS. The characteristic index characterizes the spatio-temporal interactions between input and output variables of DPS (Christofides and Daoutidis, 1996). Mathematically, the characteristic index is the smallest order of the time derivative of the controlled variable which explicitly depends on the manipulated variable. A control geometric law can be designed if the characteristic index is finite. For DPSs, the characteristic index is finite if the manipulated variable (actuator) and the controlled variable (sensor) are collocated and is infinite if they are anti-collocated. This can be explained by the number of spatial points, assuming the idea of a spatial discretization, that the manipulated variable has to go through in order to observe its effect on the controlled output.

In this section, the geometric control laws that solve the formulated control problem in Section 2 for both counter-current and co-current heat exchangers are derived.

\subsection{Control law design}

Using the characteristic index, a control law that yields a linear DPS between an external input and the controlled output can be obtained. When the characteristic index is finite, two kinds of control law are possible: the input-output linearizing and the output stabilizing control laws, which yields an unstable (chain of integrators) and a stable linear LPS, respectively. These control laws are determined separately for the counter-current and co-current heat exchangers.

\subsubsection{Counter-current heat exchanger}

The characteristic index of a linear system is obtained by considering the successive time derivatives of the controlled output until the manipulated input appears explicitly. By setting $\beta=1$ in the state 
equation (2) and evaluating the first time derivative of the controlled output (9), we obtain

$$
\begin{aligned}
\frac{d T_{c_{l}}(t)}{d t} & =\left.\frac{\partial T_{c}(z, t)}{\partial t}\right|_{z=l} \\
& =\left.\vartheta_{c} \frac{\partial T_{c}(z, t)}{\partial z}\right|_{z=l}+\alpha_{c}\left(T_{h}(l, t)-T_{c}(l, t)\right) \\
& =\left.\vartheta_{c} \frac{\partial T_{c}(z, t)}{\partial z}\right|_{z=l}+\alpha_{c}\left(T_{h_{l}}(t)-T_{c_{l}}(t)\right)
\end{aligned}
$$

As the manipulated variable $T_{h_{l}}(t)$ appears explicitly in the first derivative of $T_{c_{l}}(t)$, the characteristic index is $\sigma=1$. Note that, in the counter-current case, the manipulated and controlled variables are collocated. Solving the following equation

$$
v(t)=\left.\vartheta_{c} \frac{\partial T_{c}(z, t)}{\partial z}\right|_{z=l}+\alpha_{c}\left(T_{h_{l}}(t)-T_{c_{l}}(t)\right)
$$

with respect to the manipulated variable $T_{h_{l}}(t)$ yields the following input-output linearizing control law

$$
T_{h_{l}}(t)=\frac{v(t)+\alpha_{c} T_{c_{l}}(t)-\left.\vartheta_{c} \frac{\partial T_{c}(z, t)}{\partial z}\right|_{z=l}}{\alpha_{c}}
$$

where $v(t)$ is introduced as the external input.

Thus, in closed loop, the following linear LPS results

$$
\frac{\bar{T}_{c_{l}}(s)}{V(s)}=\frac{1}{s}
$$

where $s$ is the Laplace variable, $\bar{T}_{c_{l}}(s)$ and $V(s)$ are the Laplace transforms of $T_{c_{l}}(t)$ and $v(t)$, respectively. To obtain an output stabilizing control law, consider the following feedback control

$$
v(t)=T_{c_{l}}(t)+\gamma_{1} \frac{d T_{c_{l}}(t)}{d t}
$$

where $\gamma_{1}$ is a time parameter chosen so that the resulting closed loop system is stable. The composition of Equations (9), (12) and (16) yields the following equation

$$
v(t)=T_{c_{l}}(t)+\left.\gamma_{1} \vartheta_{c} \frac{\partial T_{c}(z, t)}{\partial z}\right|_{z=l}+\gamma_{1} \alpha_{c}\left(T_{h_{l}}(t)-T_{c_{l}}(t)\right)
$$


and by solving the resulting equation with respect to the manipulated variable $T_{h_{l}}(t)$, the following output stabilizing feedback results

$$
T_{h_{l}}(t)=\frac{v(t)+\left(\gamma_{1} \alpha_{c}-1\right) T_{c_{l}}(t)-\left.\gamma_{1} \vartheta_{c} \frac{\partial T_{c}(z, t)}{\partial z}\right|_{z=l}}{\gamma_{1} \alpha_{c}}
$$

which yields the following closed loop first order transfer function

$$
\frac{\bar{T}_{c_{l}}(s)}{V(s)}=\frac{1}{\gamma_{1} s+1}
$$

\subsubsection{Co-current heat exchanger}

In the case of the co-current heat exchanger, the manipulated and controlled variables are non-collocated, opposite to the counter-current heat exchanger where they were collocated, thus the characteristic index is infinite (Maidi et al., 2010). To design the geometric control law, we use the approach proposed by Maidi and Corriou (2011a) that consists in the following three steps:

1. Define an auxiliary controlled output $T_{c}^{m}(t)$ as the spatial weighed average temperature of the cold fluid, that is,

$$
\begin{aligned}
& T_{c}^{m}(t)=\mathcal{C} T(z, t) \\
& =\int_{0}^{l}\left[\begin{array}{ll}
0 & c(z)
\end{array}\right] T(z, t) d z \\
& =\int_{0}^{l} c(z) T_{c}(z, t) d z
\end{aligned}
$$

where $\mathcal{C}$ is a linear bounded operator and $c(z)$ is a smooth shaping function that satisfies $c(0) \neq 0$.

2. Insert the manipulated variable $T_{h_{0}}(t)$ in the state equation (1) using the notion of the extended operator (Stafford and Dowrick, 1977). It allows to convert the boundary control problem into a punctual one.

3. Derive the geometric feedback to control the auxiliary controlled output $T_{c}^{m}(t)$ using the equivalent punctual control model obtained at Step 2.

4. Define the desired reference $v(t)$ of the auxiliary controlled output $T_{c}^{m}(t)$ using either a controller 
or the steady-state mapping between the controlled variable $T_{c_{l}}(t)$ and the auxiliary output $T_{c}^{m}(t)$.

Hence, in the case of the co-current heat exchanger, the following equivalent distributed control model can be obtained using the Laplace transform as proposed by Maidi and Corriou (2011a):

$$
\begin{aligned}
& \frac{\partial T_{h}(z, t)}{\partial t}=-\vartheta_{h} \frac{\partial T_{h}(z, t)}{\partial z}+\alpha_{h}\left(T_{c}(z, t)-T_{h}(z, t)\right)+\vartheta_{h} \delta_{\varepsilon}(z) T_{h_{0}}(t) \\
& \frac{\partial T_{c}(z, t)}{\partial t}=-\vartheta_{c} \frac{\partial T_{c}(z, t)}{\partial z}+\alpha_{c}\left(T_{h}(z, t)-T_{c}(z, t)\right)
\end{aligned}
$$

with the following new boundary conditions

$$
\begin{aligned}
& T_{h}(0, t)=0 \\
& T_{c}(0, t)=T_{c_{0}}(t)
\end{aligned}
$$

where $T_{h_{0}}(t)$ is assumed to be spanned over a small portion of the spatial domain $\Omega$ (close to the boundary $z=0$ ), that is,

$$
\delta_{\varepsilon}(z)= \begin{cases}\frac{1}{\varepsilon} & \text { for } \quad 0 \leq z \leq \varepsilon \\ 0 & \text { Otherwise }\end{cases}
$$

Thus, the evaluation of the first time derivative of the auxiliary output (22) gives

$$
\begin{aligned}
\frac{d T_{c}^{m}(t)}{d t} & =\int_{0}^{l} c(z) \frac{\partial T_{c}(z, t)}{\partial t} d z \\
& =\int_{0}^{l} c(z)\left[-\vartheta_{c} \frac{\partial T_{c}(z, t)}{\partial z}+\alpha_{c}\left(T_{h}(z, t)-T_{c}(z, t)\right)\right] d z
\end{aligned}
$$

The first time derivative (29) does not depend explicitly on the manipulated variable $T_{h_{0}}(t)$, conse- 
quently the characteristic index $\sigma$ is greater than one. Performing a second time derivative, we obtain

$$
\begin{aligned}
\frac{d^{2} T_{c}^{m}(t)}{d t^{2}}= & \int_{0}^{l} c(z) \frac{\partial}{\partial t}\left[-\vartheta_{c} \frac{\partial T_{c}(z, t)}{\partial z}+\alpha_{c}\left(T_{h}(z, t)-T_{c}(z, t)\right)\right] d z \\
= & \int_{0}^{l} c(z)\left[\vartheta_{c}^{2} \frac{\partial^{2} T_{c}(z, t)}{\partial z^{2}}+2 \vartheta_{c} \alpha_{c} \frac{\partial T_{c}(z, t)}{\partial z}-\alpha_{c}\left(\vartheta_{c}+\vartheta_{h}\right) \frac{\partial T_{h}(z, t)}{\partial z}\right. \\
& \left.+\alpha_{c}\left(\alpha_{h}+\alpha_{c}\right)\left(T_{c}(z, t)-T_{h}(z, t)\right)+\alpha_{c} \vartheta_{h} \delta_{\varepsilon}(z) T_{h_{0}}(t)\right] d z
\end{aligned}
$$

We have

$$
\int_{0}^{l} c(z) \delta_{\varepsilon}(z) d z=c(0) \neq 0
$$

hence

$$
\begin{aligned}
\frac{d^{2} T_{c}^{m}(t)}{d t^{2}}= & \int_{0}^{l} c(z)\left[\vartheta_{c}^{2} \frac{\partial^{2} T_{c}(z, t)}{\partial z^{2}}+2 \vartheta_{c} \alpha_{c} \frac{\partial T_{c}(z, t)}{\partial z}-\alpha_{c}\left(\vartheta_{c}+\vartheta_{h}\right) \frac{\partial T_{h}(z, t)}{\partial z}\right. \\
& \left.+\alpha_{c}\left(\alpha_{h}+\alpha_{c}\right)\left(T_{c}(z, t)-T_{h}(z, t)\right)\right] d z+\alpha_{c} \vartheta_{h} c(0) T_{h_{0}}(t)
\end{aligned}
$$

Notice that the manipulated variable appears explicitly in the second derivative of $T_{c}^{m}(t)$, consequently the characteristic index is $\sigma=2$. By solving the following equation expressing the external input

$$
\begin{aligned}
v(t)= & \int_{0}^{l} c(z)\left[\vartheta_{c}^{2} \frac{\partial^{2} T_{c}(z, t)}{\partial z^{2}}+2 \vartheta_{c} \alpha_{c} \frac{\partial T_{c}(z, t)}{\partial z}-\alpha_{c}\left(\vartheta_{c}+\vartheta_{h}\right) \frac{\partial T_{h}(z, t)}{\partial z}\right. \\
& \left.+\alpha_{c}\left(\alpha_{h}+\alpha_{c}\right)\left(T_{c}(z, t)-T_{h}(z, t)\right)\right] d z+\alpha_{c} \vartheta_{h} c(0) T_{h_{0}}(t)
\end{aligned}
$$

with respect to the manipulated variable $T_{h_{0}}(t)$, we obtain the following input-output linearizing feedback

$$
T_{h_{0}}(t)=\frac{v(t)-\int_{0}^{l} c(z)\left[a \frac{\partial^{2} T_{c}(z, t)}{\partial z^{2}}+b \frac{\partial T_{c}(z, t)}{\partial z}-c \frac{\partial T_{h}(z, t)}{\partial z}+d \Delta T(z, t)\right] d z}{\alpha_{c} \vartheta_{h} c(0)}
$$

with $a=\vartheta_{c}^{2}, b=2 \vartheta_{c} \alpha_{c}, c=\alpha_{c}\left(\vartheta_{c}+\vartheta_{h}\right), d=\alpha_{c}\left(\alpha_{h}+\alpha_{c}\right)$ and $\Delta T(z, t)=T_{c}(z, t)-T_{h}(z, t)$. 
The state feedback (35) yields the following closed loop system:

$$
\frac{\bar{T}_{c}^{m}(s)}{V(s)}=\frac{1}{s^{2}}
$$

To obtain the output stabilizing feedback, as the characteristic index $\sigma=2$, we consider the following feedback

$$
v(t)=T_{c}^{m}(t)+\gamma_{1} \frac{d T_{c}^{m}(t)}{d t}+\gamma_{2} \frac{d^{2} T_{c}^{m}(t)}{d t^{2}}
$$

Combining Equations (29), (33) and (37), the following output stabilizing feedback follows

$$
T_{h_{0}}(t)=\frac{v(t)-T_{c}^{m}(t)-\int_{0}^{l} c(z)\left[a \frac{\partial^{2} T_{c}(z, t)}{\partial z^{2}}+b \frac{\partial T_{c}(z, t)}{\partial z}-c \frac{\partial T_{h}(z, t)}{\partial z}+d \Delta T(z, t)\right] d z}{\gamma_{2} \alpha_{c} \vartheta_{h} c(0)}
$$

with $a=\gamma_{2} \vartheta_{c}^{2}, b=\vartheta_{c}\left(2 \gamma_{2} \alpha_{c}-\gamma_{1}\right), c=\gamma_{2} \alpha_{c}\left(\vartheta_{c}+\vartheta_{h}\right)$ and $d=\alpha_{c}\left(\gamma_{2}\left(\alpha_{h}+\alpha_{c}\right)-\gamma_{1}\right)$. The state feedback (38) yields the following closed loop second order transfer function

$$
\frac{\bar{T}_{c}^{m}(s)}{V(s)}=\frac{1}{\gamma_{2} s^{2}+\gamma_{1} s+1}
$$

In the following, we focus on the output stabilizing state feedbacks, which are more practical because they yield a closed loop system, which is externally stable.

\subsection{Stability of the closed loop}

The stability of transfer functions (19) and (39), by an adequate choice of the tuning parameters $\gamma_{1}$ and $\gamma_{2}$, is not sufficient to ensure the stability of the resulting closed loop system because there is an internal dynamics related to the unobservable part of the controlled outputs. In the following, we demonstrate that, as the heat exchanger is a minimum-phase system, the internal dynamics of the resulting closed loop system is exponentially stable. In the following, we denote by $u(t)$ and $y(t)$ the manipulated and controlled variables, respectively. Thus, in the case of counter-current heat exchanger, $u(t)=T_{h_{l}}(t)$ and $y(t)=T_{c_{l}}(t)$ while in the case of co-current heat exchanger $u(t)=T_{h_{0}}(t)$ and $y(t)=T_{c}^{m}(t)$. 
Noting

$$
T(z, t)=\left[\begin{array}{c}
T_{h}(z, t) \\
T_{c}(z, t)
\end{array}\right]
$$

the models of counter-current and co-current heat exchangers can be written under the following abstract form (Curtain and Zwart, 1995)

$$
\frac{\partial T(z, t)}{\partial t}=\mathcal{A} T(z, t)+\mathcal{B} u(t)
$$

where the state operator $\mathcal{A}$ is defined as follows

$$
\mathcal{A}=\left[\begin{array}{cc}
-\vartheta_{h} & 0 \\
0 & \beta \vartheta_{c}
\end{array}\right] \frac{\partial}{\partial z}+\left[\begin{array}{rr}
-\alpha_{h} & \alpha_{h} \\
\alpha_{c} & -\alpha_{c}
\end{array}\right]
$$

and the control operator $\mathcal{B}$ is given as

$$
\mathcal{B}=\left[\begin{array}{c}
\vartheta_{h}\left(\frac{(1-\beta)}{2} \delta_{\varepsilon}(z)-\frac{(1+\beta)}{2} \delta_{\varepsilon}(l-z)\right) \\
0
\end{array}\right]
$$

with

$$
\delta_{\varepsilon}(l-z)= \begin{cases}\frac{1}{\varepsilon} & \text { for } \quad l-\varepsilon \leq z \leq l \\ 0 & \text { Otherwise }\end{cases}
$$

and $\delta_{\varepsilon}(z)$ is given by $(27)$.

Both state feedbacks (18) and (38) can be written under the following form

$$
u(t)=\mathcal{K} T(z, t)+\mathcal{L} e(t)
$$


where $\mathcal{K}$ and $\mathcal{L}$ are spatial operators that can be easily identified from the state feedback, and $e(t)$ denotes the tracking error, that is, $e(t)=v(t)-y(t)$.

Substituting the state feedback (45) into (41), the following closed loop system results

$$
\begin{aligned}
\frac{\partial T(z, t)}{\partial t} & =\mathcal{A} T(z, t)+\mathcal{B}(\mathcal{K} T(z, t)+\mathcal{L} e(t)) \\
T_{h}\left(\frac{(1+\beta)}{2} l, t\right) & =0 \\
T_{c}(0, t) & =T_{c_{0}}(t) \\
T_{h}(z, 0) & =T_{h}^{*}(z) \\
T_{c}(z, 0) & =T_{c}^{*}(z)
\end{aligned}
$$

and can be written as the interconnection of two subsystems as follows

$$
\begin{aligned}
\frac{d y(t)}{d t} & =-\frac{1}{\gamma_{1}} y(t)+\frac{1}{\gamma_{1}} v(t) \\
\frac{\partial T(z, t)}{\partial t} & =(\mathcal{A}+\mathcal{B} \mathcal{K}) T(z, t)+\mathcal{B} \mathcal{L} e(t) \\
T_{h}\left(\frac{(1+\beta)}{2} l, t\right) & =0 \\
T_{c}(0, t) & =T_{c_{0}}(t) \\
T_{h}(z, 0) & =T_{h}^{*}(z) \\
T_{c}(z, 0) & =T_{c}^{*}(z)
\end{aligned}
$$

By zeroing the auxiliary output $\left(T_{c}^{m}(t)=0\right)$ (Christofides and Daoutidis, 1996), the following zero dynamics results

$$
\begin{aligned}
\frac{\partial T(z, t)}{\partial t} & =(\mathcal{A}+\mathcal{B} \mathcal{K}) T(z, t) \\
T_{h}\left(\frac{(1+\beta)}{2} l, t\right) & =0 \\
T_{c}(0, t) & =T_{c_{0}}(t) \\
T_{h}(z, 0) & =T_{h}^{*}(z) \\
T_{c}(z, 0) & =T_{c}^{*}(z)
\end{aligned}
$$


Now, as the counter-current heat exchanger is a minimum-phase system, we conclude that the operator $\mathcal{A}+\mathcal{B} \mathcal{K}$ generates a stable $C_{0}$-semigroup $\mathcal{U}(t)$ (Christofides and Daoutidis, 1996), consequently (Pazy, 1983)

$$
\|\mathcal{U}(t)\|_{\mathcal{H}} \leq M_{T} e^{-\omega_{T} t}, \quad M_{T} \geq 1, \omega_{T}>0
$$

As the state feedback (45) yields a stable $v(t)-y(t)$ system, hence for a bounded external input $v(t)$, we have

$$
|e(t)| \leq M_{e} e^{-\omega_{e} t}, \quad M_{e} \geq 1, \omega_{e}>0
$$

where $|$.$| is the Euclidean norm.$

Thus, from the $C_{0}$-semigroup theory, as the tracking error $e(t) \in L^{1}\left(\mathbb{R}^{+}\right)$, the internal dynamics admits the following mild solution (Curtain and Zwart, 1995)

$$
T(z, t)=\mathcal{U}(t) T(z, 0)+\int_{0}^{t} \mathcal{U}(t-\zeta) \mathcal{B} \mathcal{L} e(\zeta) d \zeta
$$

Thereafter

$$
\begin{aligned}
\|T(z, t)\|_{\mathcal{H}} & =\left\|\mathcal{U}(t) T(z, 0)+\int_{0}^{t} \mathcal{U}(t-\zeta) \mathcal{B} \mathcal{L} e(\zeta) d \zeta\right\|_{\mathcal{H}} \\
& \leq\|T(z, 0) \mathcal{U}(t)\|_{\mathcal{H}}+\left\|\int_{0}^{t} \mathcal{U}(t-\zeta) \mathcal{B} \mathcal{L} e(\zeta) d \zeta\right\|_{\mathcal{H}} \\
& \leq\|T(z, 0)\|_{\mathcal{H}} M_{T} e^{-\omega_{T} t}+\int_{0}^{t} M_{T} e^{-\omega_{T}(t-\zeta)}\|\mathcal{B} \mathcal{L}\|_{\mathcal{H}} M_{e} e^{-\omega_{e} \zeta} d \zeta \\
& \leq\|T(z, 0)\|_{\mathcal{H}} M_{T} e^{-\omega_{T} t}+M_{T} M_{e}\|\mathcal{B} \mathcal{L}\|_{\mathcal{H}} e^{-\omega_{T} t} \int_{0}^{t} e^{\left(\omega_{T}-\omega_{e}\right) \zeta} d \zeta
\end{aligned}
$$

Now, in the case of $\omega_{x}=\omega_{e}$, it follows that

$$
\|T(z, t)\|_{\mathcal{H}} \leq M_{T}\left(\|T(z, 0)\|_{\mathcal{H}}+M_{e}\|\mathcal{B} \mathcal{L}\|_{\mathcal{H}} t\right) e^{-\omega_{T} t}
$$

and if $\omega_{x} \neq \omega_{e}$, we have

$$
\begin{aligned}
\|T(z, t)\|_{\mathcal{H}} & \leq\|T(z, 0)\|_{\mathcal{H}} M_{T} e^{-\omega_{T} t}+\frac{M_{T} M_{e}\|\mathcal{B} \mathcal{L}\|_{\mathcal{H}}}{\omega_{T}-\omega_{e}}\left(e^{-\omega_{e} t}-e^{-\omega_{T} t}\right) \\
& \leq M_{T}\left(\|T(z, 0)\|_{\mathcal{H}}+\frac{M_{e}\|\mathcal{B} \mathcal{L}\|_{\mathcal{H}}}{\left|\omega_{T}-\omega_{e}\right|}\right) e^{-\omega t}
\end{aligned}
$$


where $\omega=\min \left\{\omega_{T}, \omega_{e}\right\}$.

Hence, from relations (69) and (71), it can be concluded that the internal dynamics of the closed loop system is exponentially stable.

\subsection{Practical implementation issues}

\subsubsection{State estimation}

The control laws presented in Subsection 3.1 are of distributed nature (infinite dimensional), that is, for practical implementation, the whole state must be available. Practically, due to its infinite dimension, the whole state cannot be measured. Consequently, an observer must be designed to reconstruct the entire state from the accessible measurements (Hidayat et al., 2011). Before attempting to design an observer for a heat exchanger, the analysis of the observability property is the primary question. This property depends on the number of sensors and their locations (Ray, 1989; Waldraff et al., 1998). Observability can be analyzed using either the approximate model (indirect method) or the PDEs model (direct method) (Ray, 1989; Sano, 2007; Singh and Hahn, 2007). Note that indirect methods can mask this property and must be applied with care because they may lead to erroneous conclusions (Ray, 1989; Singh and Hahn, 2007). For the counter-current and co-current heat exchangers, both inlet and outlet temperatures are accessible for measurement. According to the studies conducted in the literature, using the PDEs model (direct approach), it is shown that, by sensing these temperatures, the observability condition is satisfied (Sano, 2007).

For observer design, both direct and indirect approaches can be used (Hidayat et al., 2011). Note that even if the direct approach using the PDE model is recommended, an approximation method must be used for practical implementation. Nevertheless, attention must be paid to the approximation method used so that the observability condition must be preserved (Ray, 1989; Singh and Hahn, 2007). In this chapter, the Kalman observer is used to reconstruct the entire temperatures of both cold and hot fluids. Thus, using the method of lines (Van de Wouwer et al., 2004), based on the finite difference scheme,

and by considering $N$ discretization points, the infinite dimensional state equations (1) and (2) of the 
heat exchanger can be approximated by the following finite dimensional state equation

$$
\begin{aligned}
\dot{T}_{\Delta}(t) & =A T_{\Delta}(t)+B u(t)+E T_{c_{0}}(t) \\
y(t) & =C x(t)
\end{aligned}
$$

where the state vector is defined according to the type of the heat exchanger as follows:

- For the counter-current heat exchanger:

$$
T_{\Delta}(t)=\left[T_{h_{0}}(t) T_{h_{2}}(t) \ldots T_{h_{N-1}}(t) T_{c_{1}}(t) T_{c_{2}}(t) \ldots T_{c_{N}}(t)\right]^{T}
$$

- For the co-current heat exchanger:

$$
T_{\Delta}(t)=\left[T_{h_{1}}(t) T_{h_{2}}(t) \ldots T_{h_{N}}(t) T_{c_{1}}(t) T_{c_{2}}(t) \ldots T_{c_{N}}(t)\right]^{T}
$$

where $T_{h_{i}}(t)$ and $T_{c_{i}}(t)(i=1, \ldots, N)$ are the temperatures of the hot and cold fluids temperatures at the position $z=i \Delta z$ with $\Delta z=l / N$ the discretization step. $A \in \mathbb{R}^{2 N \times 2 N}, B \in \mathbb{R}^{2 N \times 1}, E \in \mathbb{R}^{2 N \times 1}$ and $C \in \mathbb{R}^{1 \times 2 N}$ are the state, control, disturbance and observation matrices, respectively. These matrices depend both on the used finite difference scheme and the type of the heat exchanger. More details about the discretization of both heat exchangers can be found in Corriou (2018).

In the presence of both process and measurement noises, the approximate model of the heat exchanger is given as follows

$$
\begin{aligned}
\dot{T}_{\Delta}(t) & =A T_{\Delta}(t)+B u(t)+E T_{c_{0}}(t)+\zeta(t) \\
y(t) & =C T_{\Delta}(t)+\eta(t)
\end{aligned}
$$

where $\zeta(t)$ is the process noise and $\eta(t)$ is the measurement noises with zero mean. $Q$ and $R$ are the covariance of $\zeta(t)$ and $\eta(t)$, respectively.

As the heat exchanger is continuous and the measurements are in discrete form, we use the discrete- 
continuous (Corriou, 2018) formulation of the Kalman filter

$$
\begin{aligned}
\dot{\hat{T}}_{\Delta}(t) & =A \hat{T}_{\Delta}(t)+B u(t)+E T_{c_{0}}(t) \\
\dot{P}(t) & =A P(t)+P(t) A^{T}+Q
\end{aligned}
$$

where $P(t)$ is the covariance matrix.

The estimate $\hat{T}_{\Delta}(t)$ of the state $T_{\Delta}(t)$ from the available measurements $T_{c_{0}}(t)$ (measured disturbance) and $y(t)$ is provided by the Kalman filter in two phases (Corriou, 2018):

Prediction phase: The equations (78) and (79) are integrated from time $t=k-1$ to $t=k$ to obtain the estimate $\hat{T}_{\Delta}(k \mid k-1)$ and $\hat{P}(k \mid k-1) . \hat{T}_{\Delta}(k \mid k-1)$ being the estimate of $T_{\Delta}(t)$ at $t=k$ from the measurement at $t=k-1$.

Correction phase: The estimate obtained in the prediction phase for $t=k$ is corrected and the covariance matrix $P(t)$ is updated as follows:

$$
\begin{aligned}
\hat{T}_{\Delta}(k \mid k) & =\hat{T}_{\Delta}(k \mid k-1)+P(k \mid k-1) C^{T}\left(C P(k \mid k-1) C^{T}+R\right)^{-1}\left(y(k)-C \hat{T}_{\Delta}(k \mid k-1)\right) \\
P(k \mid k) & =\left(I-K_{k} C\right) P(k \mid k-1)
\end{aligned}
$$

Note that, in the case of co-current heat exchanger, the auxiliary controlled output $T_{c}^{m}(t)$, given by Equation (22), can be evaluated at $t=k$ as follows

$$
T_{c}^{m}(t)=\mathcal{C}_{\Delta} \hat{T}_{\Delta}(t)
$$

with $\hat{T}_{\Delta}(t) \equiv \hat{T}_{\Delta}(k \mid k)$ and the row vector $\mathcal{C}_{\Delta}$ depends on the quadrature used to approximate the integral operator $\mathcal{C}$ according to (Equation $(22)$.

\subsubsection{Robustness}

The control laws designed in Subsection 3.1 for both heat exchangers are sensitive to modeling errors and parameter uncertainties. For robustness and for stabilization, in the case of input-output linearizing control laws (14) and (35), we use the global linearizing control structure (Corriou, 2018; Kravaris and 
Kantor, 1990b) where the external input $v(t)$ is defined by means of a robust controller as follows:

- Case of the counter-current heat exchanger:

$$
v(t)=\int_{0}^{t} G_{c}(t-\xi)\left(T_{c_{l}}^{d}(\xi)-T_{c_{l}}(\xi)\right) d \xi
$$

- Case of the co-current heat exchanger:

$$
v(t)=\int_{0}^{t} G_{c}(t-\xi)\left(T_{c}^{m^{d}}(\xi)-T_{c}^{m}(\xi)\right) d s
$$

where $T_{c_{l}}^{d}(t)$ and $T_{c}^{m^{d}}(t)$ are desired references for $T_{c_{l}}(t)$ and $T_{c}^{m}(t)$, respectively, and the kernel $G_{c}(t)$ characterizes the desirable dynamical behavior for the overall control system.

Recall that, in the case of the co-current heat exchanger, the developed control laws (35) and (38) force the auxiliary output to track the desired reference $T_{c}^{m^{d}}(t)$. To solve the initial control problem formulated in Section 2, we propose to define the desired reference $T_{c}^{m^{d}}(t)$ by an external robust controller as follows

$$
T_{c}^{m^{d}}(t)=\int_{0}^{t} G_{c}(t-\xi)\left(T_{c_{l}}^{d}(\xi)-T_{c_{l}}(\xi)\right) d \xi
$$

The control strategies for the counter-current and co-current heat exchangers are summarized in Figures 2 and 3 .

\section{Closed-loop performance evaluation}

In this Section, the performances of the global linearizing control structures based on the output stabilizing state feedback are evaluated through numerical simulation for both counter-current and co-current heat exchangers. Both the external input $v(t)$ and the desired reference $T_{c}^{m^{d}}(t)$ are defined by means of a PI controller, that is,

$$
G_{c}(s)=k_{c}\left(1+\frac{1}{\tau_{i} s}\right)
$$

and their parameters $k_{c}$ and $\tau_{i}$ are tuned using the Graham and Lathrop method that minimizes the integral of time-weighted absolute error (ITAE) criterion (Corriou, 2018).

The closed loop system is simulated using the method of lines (Van de Wouwer et al., 2004), based 
on the finite difference scheme, by considering $N=100$ discretization points. For state estimation, the Kalman filter is designed using the approximate LPS model obtained by the method of lines. The integral terms involved in the control laws are evaluated using the trapezoidal quadrature. The different parameters used in simulation runs are summarized in Table 2.

The initial conditions $T_{h}^{*}(z)$ and $T_{c}^{*}(z)$ are the steady-state spatial temperature profiles obtained for an inlet cold fluid temperature equal to $25^{\circ} \mathrm{C}$ and inlet hot fluid temperature equal to $50^{\circ} \mathrm{C}$. The used measurements $T_{c_{0}}(t)$ and $y(t)$ are assumed to be corrupted with Gaussian white noise signals of standard deviation equal to $0.2^{\circ} \mathrm{C}$.

Both output tracking and disturbance rejection performances have been evaluated. The inlet temperature of the cold fluid $T_{c_{0}}(t)$ is assumed as a disturbance. The simulation test consists in applying two step changes $T_{c}^{d}(t)=120^{\circ} \mathrm{s}$ and $T_{c}^{d}(t)=60^{\circ} \mathrm{C}$ at $t=1 \mathrm{~s}$ and $t=10 \mathrm{~s}$, respectively for the countercurrent heat exchanger while for the co-current heat exchanger they are applied at $t=1 \mathrm{~s}$ and $t=40 \mathrm{~s}$, respectively. Between these steps changes, a step of $100 \%$ and of $-10 \%$ of the temperature of the inlet cold fluid $T_{c_{0}}(t)$ is imposed as an external disturbance at $t=5 \mathrm{~s}$ and at $t=20 \mathrm{~s}$ for the counter-current and co-current heat exchangers, respectively. To avoid sudden fluctuations of the manipulated variable due to the brutal set points change, the imposed set point is smoothed by a first order filter with a time constant equal to $0.2 \mathrm{~s}$.

In the simulation runs, the effect of the measurements errors on the quality of the estimation is minimized by a simple filtering using a moving average filter with a forgetting factor equal to 0.96 (Corriou, 2018). Also, the manipulated variable is held constant over a sampling period $\Delta t=0.02 \mathrm{~s}$.

The obtained results are depicted in Figures 4-5 and Figures 6-7 for the counter-current and cocurrent heat exchangers, respectively. It can be seen that the output tracking is achieved in a satisfactory manner. But in the case of co-current heat exchanger, the controlled output $T_{c_{l}}(t)$ presents slight fluctuations around the imposed set points. This is due of course to the effect of the noise measurements. Indeed, by comparing the quality of the control moves given by Figures 5 and 7 , it follows that in the case of the co-current heat exchanger, the quality of the control is more sensitive to noisy measurements. This is expected because the state feedback (38) is more sensitive to the measurement noise since it involves more spatial derivatives compared to the state feedback (18). Note that without noise measurements, perfect tracking is achieved for both heat exchangers.

Also, from the results obtained, it can be observed that the disturbance effect is damped, which 
demonstrates the disturbance rejection capability of both control strategies. Notice that, in the case of the counter-current heat exchanger (collocated case), the effect of the disturbance can be observed on the controlled output only if the disturbance is important. On another side, the disturbance effect is attenuated as the observation position moves and the effect becomes nearly invisible at the outlet of the heat exchanger (Maidi et al., 2009).

\section{Conclusion}

Based on the geometric control theory, a state feedback of infinite dimensional nature is designed both for counter-current and co-current heat exchangers to control the outlet temperature of the cold fluid by manipulating the inlet temperature of the hot fluid. The design approach is based on the PDE model. Two kinds of state feedback are developed: input-output linearizing and output stabilizing control laws. In the case of the counter-current heat exchanger, it is shown that, as the actuator and the sensor are collocated, the characteristic index is finite and the control laws can be designed easily by evaluating the first time derivative of the outlet. In the case of the co-current heat exchanger, the characteristic index is infinite because the sensor and the actuator are non-collocated, so that a different design approach consisting in four steps is presented. The idea consists in introducing the manipulated variable in the state equation using the notion of the extended operator, and then a control law is designed by assuming an auxiliary output, defined as the spatial average temperature of the cold temperature, instead of its outlet temperature. To achieve the original control problem, a control strategy is presented where the desired reference for the auxiliary output is defined by means of an external controller. The robustness, the stability of the resulting closed loop systems and the implementation of the developed control laws issues are discussed. The tracking and disturbance rejection performances are evaluated via numerical simulation for both counter-current and co-current heat exchangers.

Notice that the direct design approaches, presented in this chapter, can be adopted to design infinite dimensional boundary controllers for $2 \times 2$ hyperbolic systems of balance laws. 


\section{References}

M. A. Abdelghani-Idrissi, M. A. Arbaoui, L. Estel, and J. Richalet. Predictive functional control of a counter current heat exchanger using convexity property. Chemical Engineering and Processing: Process Intensification, 40(5):449-457, 2001.

S. K. Al-Dawery, A. M. Alrahawi, and K. M. Al-Zobai. Dynamic modeling and control of plate heat exchanger. International Journal of Heat and Mass Transfer, 55(23):6873-6880, 2012.

N. B. Almutairi and Mohamed Zribi. Control of a plate heat exchanger using the terminal sliding mode technique. Industrial \& Engineering Chemistry Research, 51(12):4610-4623, 2012.

S. Alotaibi, M. Sen, B. Goodwine, and K. T. Yang. Controllability of cross-flow heat exchangers. International Journal of Heat and Mass Transfer, 47(5):913-924, 2004.

M. A. Arbaoui, L. Verniéres-Hassimi, D. Seguin, and M. A. Abdelghani-Idrissi. Counter-current tubular heat exchanger: Modeling and adaptive predictive functional control. Applied Thermal Engineering, 27(13):2332-2338, 2007.

K. Atkinson and W. Han. Theoretical Numerical Analysis. A Functional Analysis Framework. SpringerVerlag, New York, 2009.

E. Aulisa, J. A. Burns, and D. S. Gilliam. Velocity control of a counter-flow heat exchanger. IFACPapersOnLine, 49(18):104-109, 2016.

K. Bartecki. Modeling and Analysis of Linear Hyperbolic Systems of Balance Laws. Springer, Switzerland, 2016.

D. Bittanti and L. Piroddi. Nonlinear identification and control of a heat exchanger: A neural network approach. Journal of The Franklin Institute, 334B(1):135-153, 1997.

E. Bühler and D. Franke. Topics in Identification and Distributed Parameter Systems. Springer, Wiesbaden, 1980 .

P. D. Christofides. Nonlinear and Robust Control of PDE Systems: Methods and Applications to Transport-Reaction Processes. Birkhäuser, Boston, 2001. 
P. D. Christofides and P. Daoutidis. Feedback control of hyperbolic PDE systems. AIChE Journal, 42 (11):3063-3086, 1996.

J.-P. Corriou. Process control - Theory and applications. Springer, London, 2nd edition, 2018.

R. F. Curtain and H. Zwart. An Introduction to Infinite-Dimensional Linear Systems Theory. Springer, New York, 1995.

M. Dulău, S. Oltean, and A. Gligor. Conventional control vs. robust control on heat-exchangers. Procedia Technology, 19:534-540, 2015.

M. Fischer, O. Nelles, and R. Isermann. Adaptive predictive control of a heat exchanger based on a fuzzy model. Control Engineering Practice, 6(2):259-269, 1998.

M. Fratczak, J. Czeczot, P. Nowak, and M. Metzger. Practical validation of the effective control of liquid-liquid heat exchangers by distributed parameter balance-based adaptive controller. Applied Thermal Engineering, 129(1):549-556, 2018.

J. C. Friedly. Dynamic Behaviour of Processes. Prentice-Hall, New Jersey, 1972.

J. P. Gauthier and C. Z. Xu. $\mathcal{H}_{\infty}$ control of a distributed parameter system with non-minimum phase. International Journal of Control, 53(1):45-79, 1991.

P. Grabowski. Stability of a heat exchanger feedback control system using the circle criterion. International Journal of Control, 80(9):1388-1403, 2007.

E. M. Hanczyc and A. Palazoglu. Nonlinear control of a distributed parameter process: the case of multiple caracteristics. Industrial \& Engineering Chemistry Research, 34(12):4406-4412, 1995.

Z. Hidayat, R. Babusška, B. De Schutter, and A. Núñez. Observers for linear distributed parameter systems: A survey. In the Proceedings of the 2011 IEEE International Symposium on Robotics and Sensors Environments (ROSE 2011), Montreal, Canada, pages 166-171, September 17-18, 2011.

A. Isidori. Nonlinear control systems. Springer-Verlag, New York, 1995.

S. Kakaş and H. Liu. Heat Exchangers: Selection, Rating, and Thermal Design. CRC Press, Boca Raton, 2002. 
H. Kanoh, T. Itoh, and N. Abe. Nonlinear $\mathrm{H}_{\infty}$ control for heat exchangers controlled by the manipulation of flow rate. Nonlinear Analysis: Theory, Methods \& Applications, 30(4):2237-2248, 1997.

C. Kravaris and J. C. Kantor. Geometric methods for nonlinear process control. 1. background. Industrial 83 Engineering Chemistry Research, 29(12):2295-2310, 1990a.

C. Kravaris and J. C. Kantor. Geometric methods for nonlinear process control. 2. controller synthesis. Industrial \& Engineering Chemistry Research, 29(12):2310-2323, 1990b.

N. Kunimatsu. Stability analysis of heat-exchanger equations with boundary feedbacks. IMA Journal of Mathematical Control \& Information, 15(4):317-330, 1998.

H. X. Li and C. Qi. Modeling of distributed parameter systems for applications-A synthesized review from time-space separation. Journal of Process Control, 20(8):891-901, 2010.

A. Maidi and J.-P. Corriou. Boundary control of nonlinear distributed parameter systems by inputoutput linearization. In the Proceedings of the 18th IFAC World Congress, Milan, Italy, pages 1091010915, August 28-September 02, 2011 a.

A. Maidi and J.-P. Corriou. Distributed feedback design for systems governed by the wave equation. International Journal of Control, 84(8):1417-1429, 2011b.

A. Maidi and J.-P. Corriou. Distributed control of nonlinear diffusion systems by input-output linearization. International Journal of Robust and Nonlinear Control, 24(3):386-405, 2014a.

A. Maidi and J.-P. Corriou. Boundary control of linear stefan problem. Journal of Process Control, 24 (6):939-946, 2014b.

A. Maidi, M. Diaf, and J.-P. Corriou. Boundary geometric control of a counter-current heat exchanger. Journal of Process Control, 19(2):297-313, 2009.

A. Maidi, M. Diaf, and J.-P. Corriou. Boundary control of a parallel-flow heat exchanger by input-output linearization. Journal of Process Control, 20(10):1161-1174, 2010.

Y. S. N. Malleswararao and M. Chidambaram. Non-linear controllers of a heat exchanger. Journal of Process Control, 2(1):17-21, 1992. 
A. H. Mazinan and N. Sadati. On the application of fuzzy predictive control based on multiple models strategy to a tubular heat exchanger system. Transactions of the Institute of Measurement and Control, 32(4):395-418, 2010.

A. Pazy. Semigroups of Linear Operators and Applications to Partial Differential Equations. Springer, New York, 1983.

S. Pohjolainen and I. Lätti. Robust controller for boundary control systems. International Journal of Control, 38(6):1189-1197, 1983.

W. H. Ray. Advanced Process Control. Butterworths, Boston, 1989.

W. H. Ray and B. A. Ogunnaike. Process Dynamics, Modeling and Control. Oxford University Press, 1994.

H. Sano. Observability and reachability for parallel-flow heat exchanger equations. IMA Journal of Mathematical Control and Information, 24(1):137-147, 2007.

H. Sano. Boundary control of a parallel-flow heat exchange process with boundary observation. IFACPapersOnLine, 48(1):755-760, 2015.

H. Shang, J. F. Forbes, and M. Guay. Feedback control of hyperbolic distributed parameter systems. Chemical Engineering Science, 60(4):969-980, 2005.

A. Singh and J. Hahn. Effect of finite-dimensional approximation on observability analysis of distributed parameter models. 8th International IFAC Symposium on Dynamics and Control of Process Systems, June 6-8, Cancún, Mexico, 2007.

Z. Stafford and G. Dowrick. Applications of extended operator to diffusive systems. In Proceedings of the Second IFAC Symposium on Distributed Parameter Systems, Coventry, Great Britain, pages 525-537, 28 June-1 July, 1977.

A. Van de Wouwer, P. Saucez, and W. E. Schiesser. Simulation of distributed parameter systems using a Matlab-based method of lines toolbox: Chemical engineering applications. Industrial and Engineering Chemical Research, 43(14):3469-3477, 2004. 
A. Vasičkaninová and M. Bakošová. Control of a heat exchanger using neural network predictive control combined with auxiliary fuzzy controller. Applied Thermal Engineering, 89(10):1046-1053, 2015.

A. Vasičkaninová and M. Bakošová. Robust controller design for a heat exchanger using $\mathcal{H}_{2}, \mathcal{H}_{\infty}$, $\mathcal{H}_{2} / \mathcal{H}_{\infty}$, and $\mu$-synthesis approaches. Acta Chimica Slovaca, 9(2):184-193, 2016.

A. Vasičkaninová, M. Bakošová, A. Mészáros, and J. J. Klemeš. Neural network predictive control of a heat exchanger. Applied Thermal Engineering, 31(13):2094-2100, 2011.

A. Vasičkaninová, M. Bakošová, L. Čirka, M. Kalúz, and J. Oravec. Robust controller design for a laboratory heat exchanger. Applied Thermal Engineering, 128(1):1297-1309, 2018.

W. Waldraff, D. Dochain, S. Bourrel, and A. Magnus. On the use of observability measures for sensor location in tubular reactor. Journal of Process Control, 8(5-6):497-505, 1998.

W. Wu and C. T. Liou. Output regulation of nonisothermal plug-flow reactors with inlet perturbations. Computers and Chemical Engineering, 25(2-3):433-443, 2001. 


\begin{tabular}{|l|c|c|c|}
\cline { 2 - 4 } \multicolumn{1}{c|}{} & Manipulated variable & Controlled variable & Actuator and sensor location \\
\hline Counter-current & $T_{h_{l}}(t)$ & $T_{c_{l}}(t)$ & Collocation \\
\hline Co-current & $T_{h_{0}}(t)$ & $T_{c_{l}}(t)$ & Anti-collocation \\
\hline
\end{tabular}

Table 1: Manipulated and controlled variables for the heat exchangers.

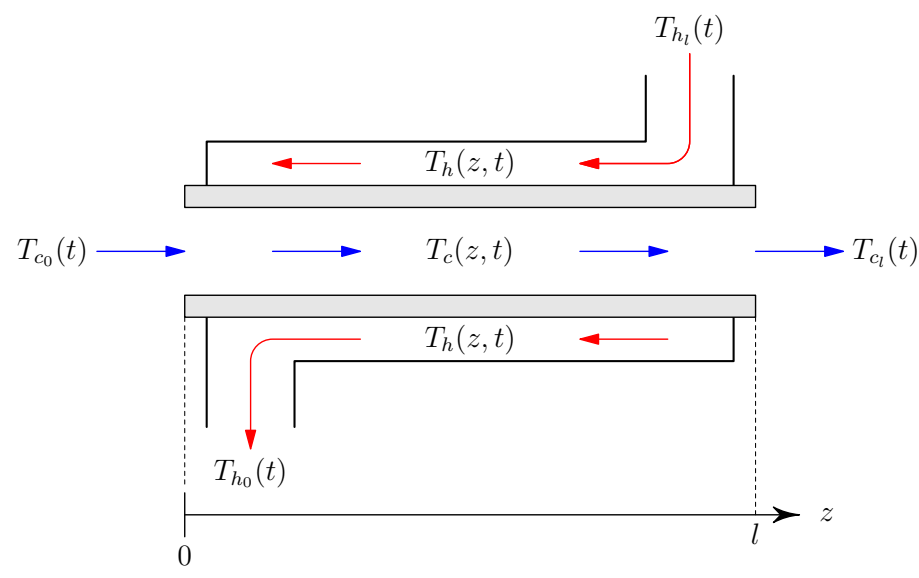

(a) Counter-current

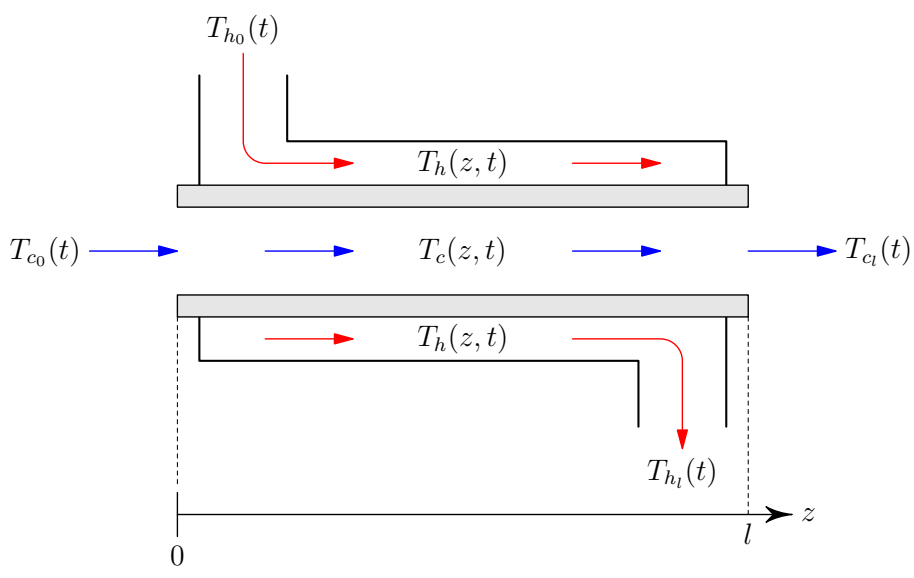

(b) Cocurrent

Figure 1: Tube heat exchangers.

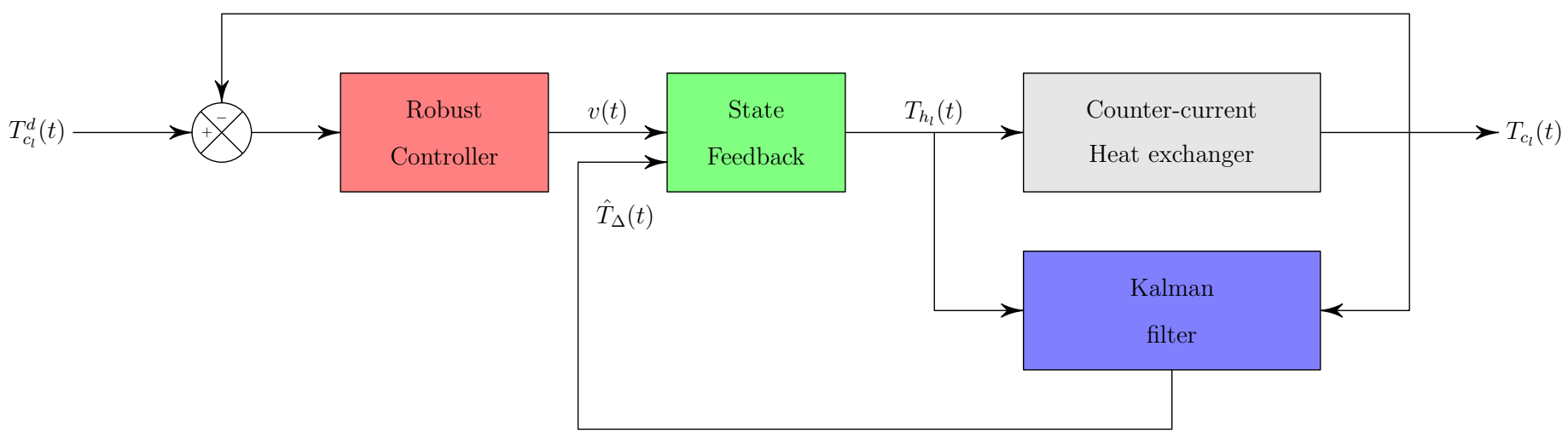

Figure 2: Global control strategy for counter-current heat exchanger.

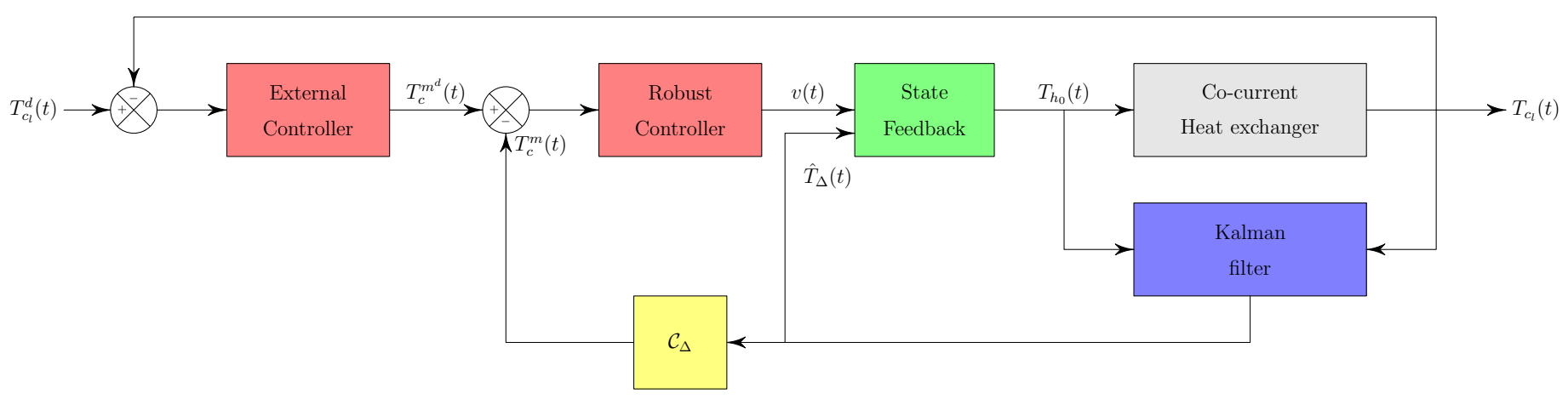

Figure 3: Global control strategy for co-current heat exchanger. 


\begin{tabular}{|c|c|c|}
\hline & Counter-current heat exchanger & Co-current heat exchanger \\
\hline Physical parameters & \multicolumn{2}{|c|}{$v_{c}=1 \mathrm{~m} \cdot \mathrm{s}^{-1}, v_{h}=2 \mathrm{~m} \cdot \mathrm{s}^{-1}, \alpha_{c}=1 \mathrm{~s}^{-1}, \alpha_{h}=1 \mathrm{~s}^{-1}$} \\
\hline Initial inlet temperature $(t=0)$ & $T_{c_{0}}=25^{\circ} \mathrm{C}, T_{h_{l}}=50^{\circ} \mathrm{C}$ & $T_{c_{0}}=25^{\circ} \mathrm{C}, T_{h_{0}}=50^{\circ} \mathrm{C}$ \\
\hline State feedback & $\gamma_{1}=1 \mathrm{~s}$ & $\gamma_{1}=0.22 \mathrm{~s}, \gamma_{2}=5 \times 10^{-2} \mathrm{~s}^{2}$ \\
\hline Robust controller & $k_{c}=7.8, \tau_{i}=1.03 \mathrm{~s}$ & $k_{c}=2.4 \times 10^{-2}, \tau_{i}=4.69 \times 10^{-2} \mathrm{~s}$ \\
\hline External controller & - & $k_{c}=2.68 \times 10^{-2}, \tau_{i}=0.3253 \mathrm{~s}$ \\
\hline Kalman filter & $P(0 / 0)=10^{-2} I_{2 N \times 2 N}, Q=5 \times 10^{-2} I_{2 N \times 2 N}, R=10^{-2}$ & $Q=P(0 / 0)=0.1 I_{2 N \times 2 N}, R=0.1 I_{2 \times 2}$ \\
\hline
\end{tabular}

Table 2: Parameters used in simulation. 


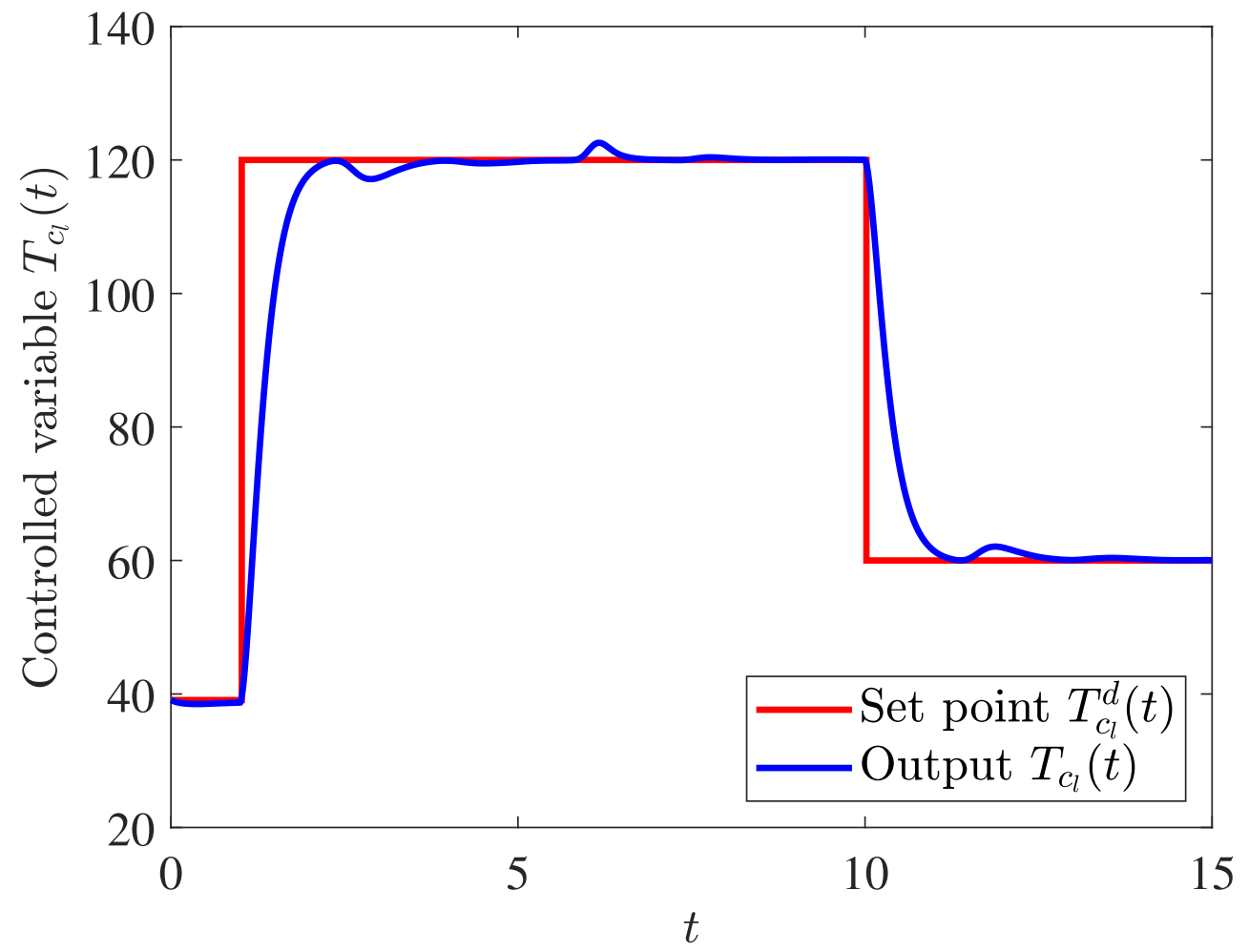

Figure 4: Counter-current heat exchanger: Evolution of the controlled output $T_{c_{l}}(t)$.

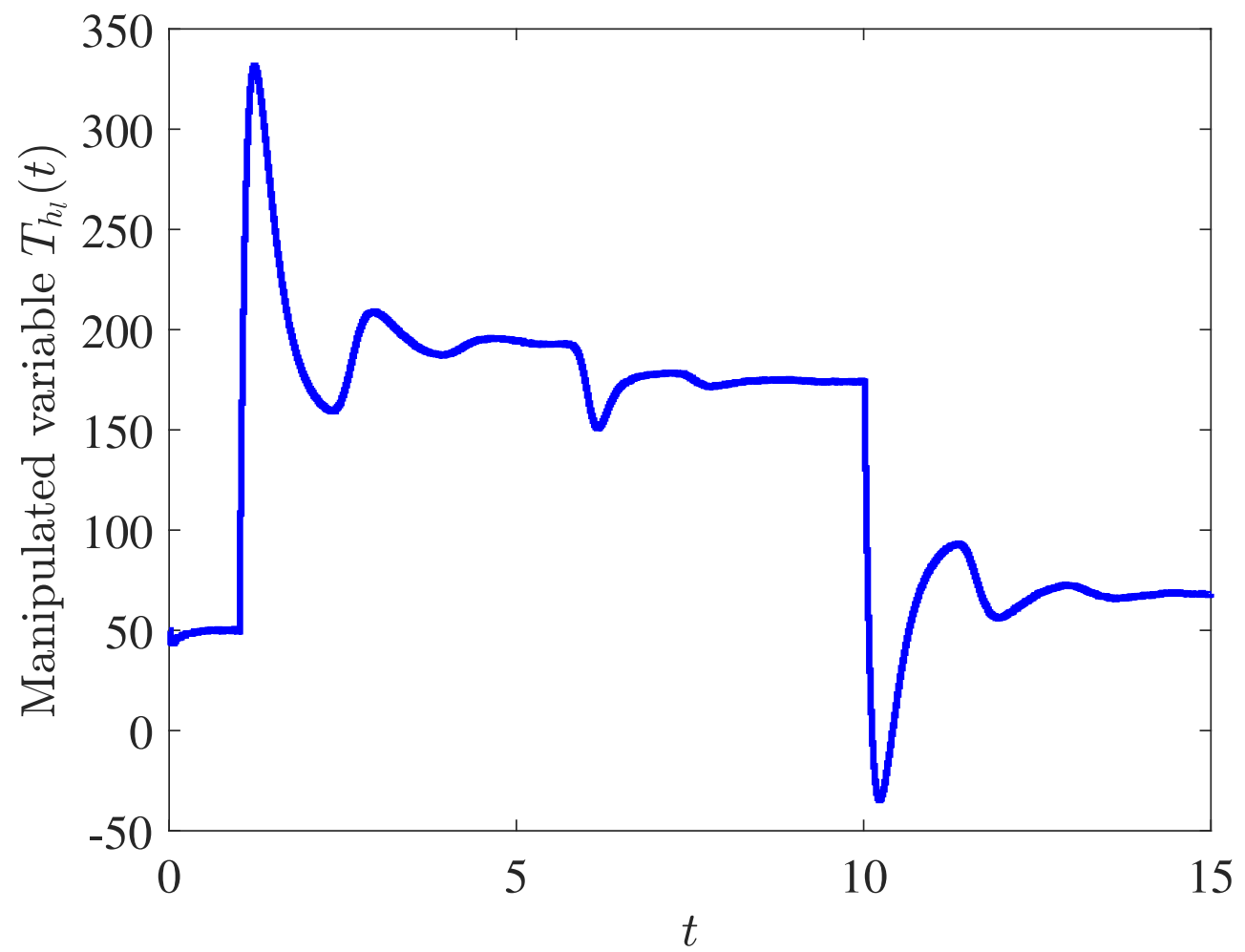

Figure 5: Counter-current heat exchanger: Evolution of the manipulated variable $T_{h_{l}}(t)$ 


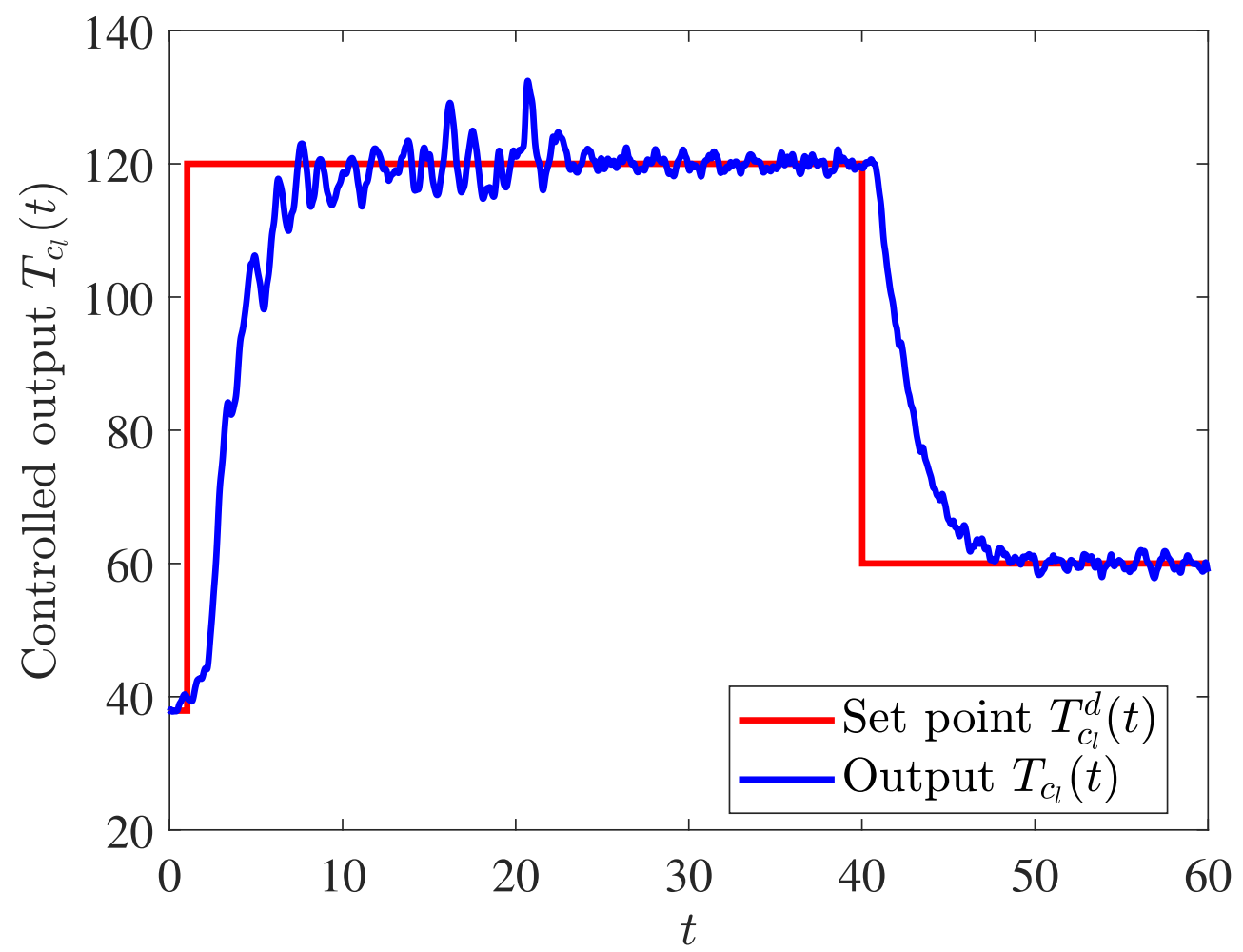

Figure 6: Co-current heat exchanger: Evolution of the controlled output $T_{c_{l}}(t)$.

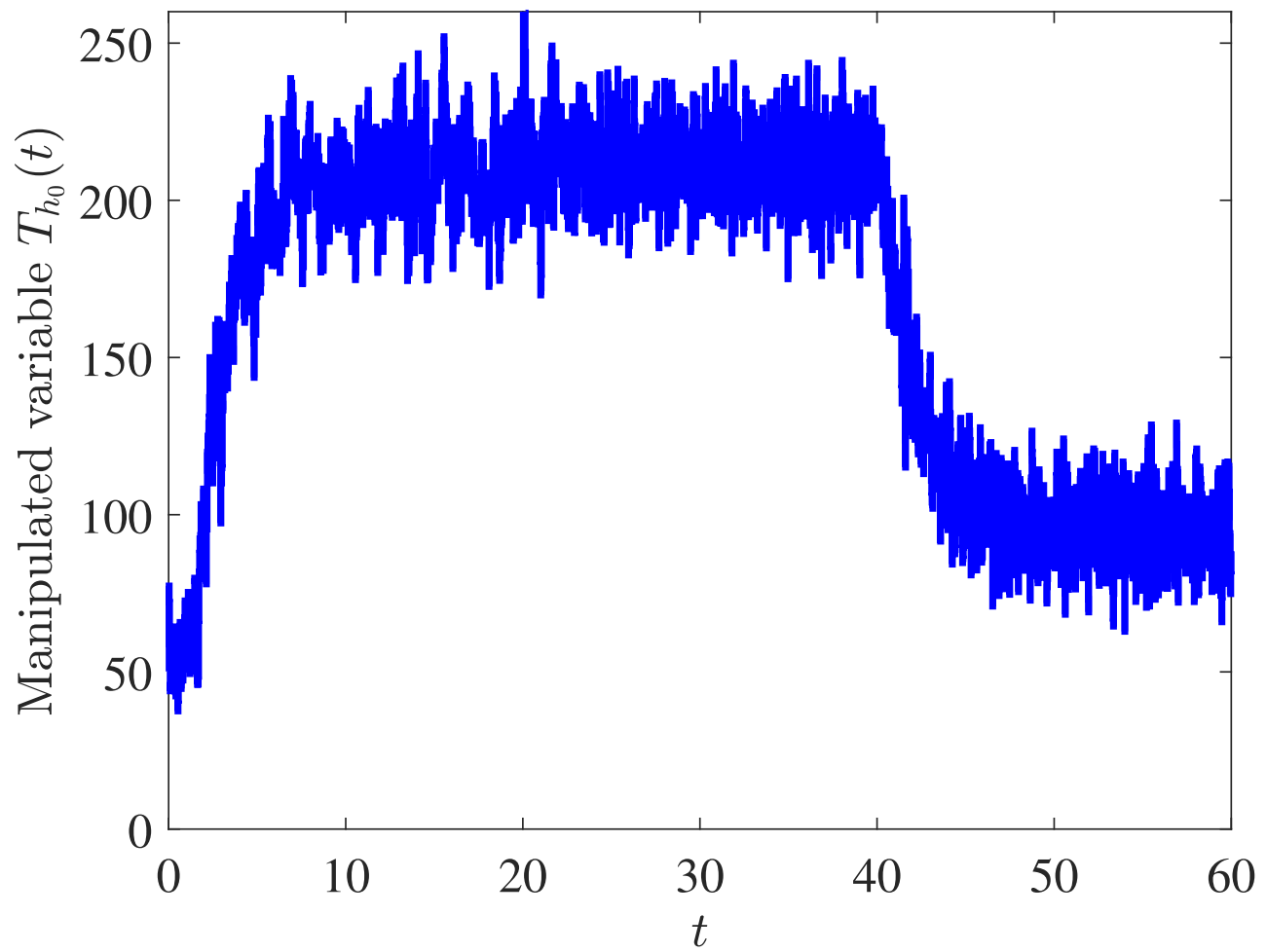

Figure 7: Co-current heat exchanger: Evolution of the manipulated variable $T_{h_{0}}(t)$. 